\title{
An Assessment Of The Health Literacy Among Deaf People In Kapiri Mposhi District Of Zambia
}

\author{
${ }^{1 *}$ Brian Chanda Chiluba, ${ }^{2}$ Mutale Chibo, Beatrice ${ }^{3}$ Mwaba-Chiluba and ${ }^{4}$ Beverly Thole \\ ${ }^{1}$ The University of Zambia, School of Health Sciences, Department of Physiotherapy, P.O. Box50110, Lusaka. \\ ${ }^{2}$ The University of Lusaka, School of Health Sciences, Department of Public Health, Lusaka. \\ ${ }^{3}$ Community Based Rehabilitation (CBR)/ Community Based Inclusive Development (CBID) P.O. Box 60080
}

Livingstone

\begin{abstract}
Health care is important to everyone and everyone should have equal access to health care. The literature available focuses mainly on high-income countries. It is therefore important to bear in mind that many Deaf people, especially in low and middle-income countries, are suffering from much greater health disparities. Deaf people rely on a visual language that does not have a written form. These individuals may lack proficiency in written English. Health literacy, as defined by the National Institutes of Health refers to how well a person can get the health information and services that they need, and how well they understand them. To assess the Health Literacy of Deaf people in Kapiri Mposhi District. This research was a qualitative study; it used a crosssectional descriptive study design. Snowball sampling was done. The sample size was 15 participants. 10 Deaf individuals and 5 health care providers altogether. Interviews were conducted and manual transcription was used to analyse the data and translate it into themes. Deaf people in Kapiri Mposhi are not aware of issues pertaining to health and wellbeing. It appears that Deaf people, because of communication barriers faced, do not receive full disclosure of their medical situation and the alternatives are not discussed with them. Even when disclosure does occur, many Deaf people may not fully comprehend the options available to them because of communication difficulties with health care providers. This entails that the Deaf people in Kapiri Mposhi District have their health literacy extremely low.
\end{abstract}

Keywords: Deaf; Health Literacy; Sign Language

\section{Introduction}

The World Health Organization's global strategy, 'Health for Al1 by the Year 2000" is based on the principle that health professionals, educators, scientists, politicians, government committees, and others must cooperate to enhance the quality of life for all people (WHO, 1981). Recently, disparate health care of people with disabilities has captured the attention of the international health and development community. The need to identify barriers that people with disabilities face in accessing health services at a variety of levels, and to find optimum strategies to integrate their needs into primary health care systems by focusing on delivering effective interventions have been highlighted as priorities.

\footnotetext{
* Corresponding author: Brian Chanda Chiluba brian.chiluba@unza.zm

Published online at http://IJDS.ub.ac.id/

Copyright $\odot$ 2014PSLD UB Publishing. All Rights Reserved
}

The literature available focuses mainly on high-income countries. It is therefore important to bear in mind that many Deaf people, especially in low and middle-income countries, are suffering from much greater health disparities. According to Richardson Health promotion and education is severely lacking among the Deaf (Richardson, 2014). This places them at unnecessary high risk for frequent development of non-communicable diseases (NCDs). Further, regular physicals and screenings are not performed due to healthcare avoidance there is also limited education available about necessary lifestyle modifications, medications, or additional treatments to manage their disease.

The Deaf population do not receive adequate education about topics such as safety, mental health, alcohol, drugs, and sex education about balanced meals and nutritional treatment of health issues is again hampered by communication barriers. Erroneous knowledge on subjects such as nutrition and high-risk 
behaviours is propagated among the Deaf without access to the factual sources (Smith et al, 2012.

\section{Research Methodology}

This research was a qualitative study using a cases study approach. The study was carried out in Kapiri Mposhi District, a town located in Central Province. Kapiri Mposhi lies on the Great North Road and is significant for the railway connection between Zambia railways line from Kitwe to Lusaka and Livingstone and western terminal of the Tanzania-Zambia railway authority from Dar es Salaam since 1976. As of 2010, the total population of Kapiri Mposhi is 14,792 and the Kapiri Mposhi District has more than 60 deaf individuals that form the deaf community. Small towns are usually overlooked when conducting Research studies, it is for this reason that Kapiri Mposhi District was picked for the purpose of this research. The study target was the Central District of Kapiri Mposhi town. The health care facilities were the target sources of respondents for the researcher. The respondents were 5 Health care professionals and 10 Deaf persons. The sample size was 15 respondents after reaching saturation. The research sampling method that was used in this study is snowball sampling method, this approach is a valuable tool in studying the lifestyles of groups and it is often used when the respondents are few in number.

For the purposes of this research, interviews were used. The interviews were conducted in American Sign Language (ASL). Since the structure of Sign Language differs significantly from English, interview questions were translated into Sign Language prior to the interviews

Video shooting was done on the Deaf individuals. Phone recording was done and note taking when interviewing the health care providers. A semi-structured interview guide was used with open-ended questions to guide participants as they related knowledge pertaining to the phenomena of health and deafness.

The process of data analysis is a creative process. It involves uncovering patterns, themes, and categories and requires ranking carefully considered judgments about what is really significant and meaningful in the data. Data analysis was one by manual transcription and arranging of data into themes. No software was used, transcribing the data began immediately during the data collection process this enabled emerging themes to be identified. All transcribed data was manually typed after listening to the audio recordings and watching the video.

\section{Results and Discussion}

Results revealed that Deaf persons do not have access to health information and that they find it challenging to communicate effectively with health care providers. One Participant Stated:

"...communication with the health workers is done though writing on paper, and it is not good because they don't explain everything..."

Additionally, misunderstandings about the expression of pain are also possible because many healthcare providers know little to nothing about the major role that facial expressions play in Deaf communication. Another participant mentioned that:

\section{“...i just point where the problem is on my body and the health worker writes the diagnosis..."}

All persons interviewed placed a great deal of importance on the attitude of health care providers. A positive attitude is generally interpreted as acceptance of the person and facilitates communication, whereas a negative attitude is often perceived as rejection and impedes communication. Negative attitudes are generally attributed to a general lack of knowledge of health professional regarding Deaf persons. For example, the healthcare provider may be perceived as impolite if they do not maintain eye contact when speaking to a Deaf person. It was brought to my attention by another participant that:

\section{"...health workers show a non caring attitude particularly when they are tired..."}

\subsection{Emerging Themes \\ 3.1.1 The Interpreter Effect}

Effective communication can be achieved through a qualified sign language interpreter. The failure for health care providers to provide qualified interpreters is disturbing, when there is 
a need for accurate and immediate communication. Consequently, Deaf people may be fearful of having to interact with health care workers who are insensitive or uneducated about the needs of the Deaf community. For many profoundly deaf persons the issue of interpreters is presented as one of necessity and efficiency and not one of disability as is the popular view.

"...my mother serves as the interpreter when we are at the health facility though it's not everything that she knows..."

\subsubsection{Changes in the Health System}

On a personal level, there is no doubt that an interpreter can play a key role in the lives of deaf persons. Where languages and ways of being differ, an interpreter can become the voice of the deaf and the link to the outside world as he/she enables and facilitates communication, therefore, making the world of the Deaf a little easier and more pleasant. In emergency health situations, an interpreter can ultimately mean survival.

"...health workers must be taught sign language so that the needs of the Deaf people are

$$
\text { met..." }
$$

A healthcare facility for the Deaf only can be an effective way to promote health for all so that their basic health needs are met.

"...i like the idea of a health care facility because my needs as a Deaf person will be met accordingly..."

\subsubsection{Health Care Providers Mode of} Communication
Writing was the most frequent method health care providers communicate with Deaf clients. It appeared that Deaf people, because of the communication barriers faced do not receive full disclosure of their medical situation and the alternatives are not discussed with them. Even when disclosure does occur, many Deaf people may not fully comprehend the options available to them because of communication difficulties with healthcare providers.

“...it is a challenge to interpret when attending to a Deaf patient, writing on paper is time consuming..."

“...i know a few basic signs but $i$ still feel as a clinical officer, $i$ am not giving the Deaf a proper service they deserve because of communication method..."

\subsubsection{Changes in the Health System}

The health care providers that were interviewed mentioned that they would like to see some changes in the health system. One of them stated as follows:

"...there should be a deliberate policy where sign language is introduced and we as health care providers are taught the basics in sign language..."

"...it would be appropriate to have posters in health facilities that are portrayed in sign language so that the Deaf people can interpret and understand in their own language..."

The Fishbone Analysis Diagram below illustrates how the findings of the study are linked to the inadequacy of health literacy.

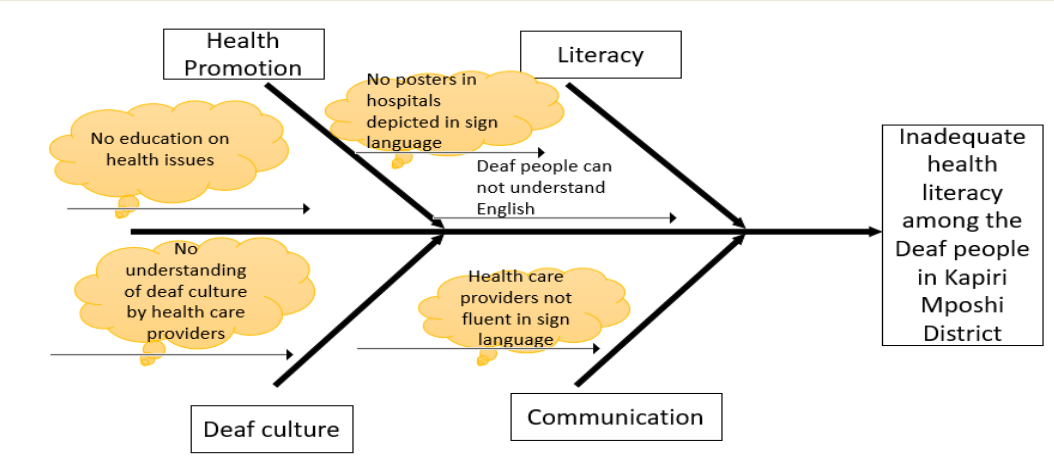

Fig 1. A Fishbone Analysis Diagram of Health Literacy among the Deaf People. 


\section{Discussion}

The objective based of the findings indicates that the Deaf people are not familiar with health information because of communication barrier between them and the Health Care Providers.

Sign Language is the natural language of deaf people, there are few health care providers conversant in sign language, and for this reason communication in a written form is utilized. Written communication is unconformable for most Deaf persons as it makes Deafness visible it discredits them because the writing skills of deaf people may not measure up to the standards set by the hearing.

In line with the findings regarding written communications a study done by Sandler et al confirms that much information is written but this is frequently inaccessible to those Deaf individuals who are limited in their ability to read English. Pamphlets and even notices often use terminology which requires a level of literacy which many Deaf individuals have not obtained (Sadler et al, 2001).

The emerging theme of lack of health knowledge was characterized by a lack of understanding resulting from inadequate communication and lack of knowledge about the realities of the deaf community, as well as poor attitudes towards deaf persons leading to an overwhelming emotional response characterized by a feeling of rejection, exclusion and powerlessness.

The findings also adequately showed that literacy is another factor adversely affecting health care for Deaf. Since English is a second language for many Deaf individuals and since they are not able to hear the language, reading can be difficult. English is based on sounds not heard by people who are deaf where as sign language depends on visual communication. Studies done by the National Institute on Deafness also revealed the similar findings (NIDOCD, 2016).

All persons interviewed placed a great deal of importance on the attitude of health professionals. A positive attitude is generally interpreted as acceptance of the person and facilitates communication, whereas a negative attitude is often perceived as rejection and impedes communication. Negative attitudes are generally attributed to a general lack of knowledge of health professionals regarding Deaf persons. Unfortunately, it appears that poor attitudes prevail. Similar studies can attest to the above finding as well (Ebert and Heckerling, 1995).

In a positive way, the people interviewed in this study all liked the emerging theme of changes to be made in the health care system. It was suggested that the Deaf would really embrace the idea of a health center for Deaf only so that their health needs are met and their potential for health is maximized.

It was noted that education materials must take literacy level into account. Since the Deaf currently exhibit serious health promotion deficits, education is needed on numerous topics. In a similar line of thought, Hammer recommended health promotion activities in the Deaf community (Hammer, 1999; Chiluba \& Njapawu, 2019).

\section{Conclusion}

This type of research is important for Deaf persons as it brings forth their voice which may contribute to breaking cultural barriers to health care.

The only way to end the vicious cycle of distrust and poor patient outcomes is to take action to promote cultural competency. Awareness must be spread especially among healthcare professionals about the details of Deaf culture in order to stop audism and promote improved, research-based care. Communication could be improved by using the recommendations which have been discussed

This study should be an eye opener because Sustainable Development Goals cannot be achieved if health for all is not reaching the vulnerable communities such as the Deaf communities. There is need therefore for deliberate policies by policy makers to include the use of sign language in the health care system.

\section{References}

National institutes of Health, (2010) U.S. Department of Health and Human Resource.

World Health Organization (1981). Global strategy for health for all by the year 2000 Geneva: WHO.

Richardson, K. (2014). Deaf culture: Competencies and best practices. The Nurse Practitioner. 
Smith, C., Massey-Stokes, M., \& Lieberth, A. (2012). Health information needs of Deaf adolescent females: A call to action. American Annals of the Deaf

Sadler GR, Huang JT, Padden CA, Elion L, Galey TA, Gunsauls DC, Brauer B (2001)

Bringing Health Care information to the Deaf Community. Journal of Cancer Education, 16, pp.105-108.

National Institute on Deafness and Other Communication Disorders. (2016). Quick statistics about hearing.
Ebert DA and Heckerling PS (1995) Communication with Deaf patients: knowledge, beliefs and practices of physicians. Journal of the American Medical Association, 273(3), pp.227-229.

Harmer (1999). Health care delivery and Deaf people; Practice, problems and recommendations for change. Journal of Deaf studies and Deaf education.

Chiluba BC \& Njapawu WG (2019). Barriers of Persons with Physical Disability over Accessibility and Mobility to Public Buidlings in Zambia. Indonesian Journal of Disability Studies(IJDS). 2019: Vol. 6 (1):PP 53-63. 